\title{
Path monotonicity, consistency and axiomatizations of some weighted solutions
}

\author{
Pedro Calleja · Francesc Llerena
}

Received: date / Accepted: date

\begin{abstract}
On the domain of cooperative games with transferable utility, we introduce path monotonicity, a property closely related to fairness (van den Brink, 2001). The principle of fairness states that if a game changes by adding another game in which two players are symmetric, then their payoffs change by the same amount. Under efficiency, path monotonicity is a relaxation of fairness that guarantees that when the worth of the grand coalition varies, the players' payoffs change according to some monotone path. In this paper, together with the standard properties of projection consistency (Funaki, 1998) and covariance, we show that path monotonicity characterizes the weighted surplus division solutions. Interestingly, replacing projection consistency by either self consistency (Hart and Mas-Colell, 1989) or max consistency (Davis and Maschler, 1965) we obtain new axiomatic characterizations of the weighted Shapley values and the prenucleolus, respectively. Finally, by the duality approach we provide a new axiomatization of the weighted egalitarian non-separable contribution solutions using complement consistency (Moulin, 1985).
\end{abstract}

Keywords consistency · weighted surplus division solutions · weighted egalitarian non-separable contribution solutions · weighted Shapley values · prenucleolus

JEL classification: Cr71, $^{7} 78$

P. Calleja

Dep. de Matemàtica Econòmica, Financera i Actuarial and BEAT, Universitat de Barcelona, Av. Diagonal, 690, 08034 Barcelona, Spain

E-mail: calleja@ub.edu

F. Llerena

Dep. de Gestió d'Empreses, Universitat Rovira i Virgili and CREIP, Av. de la Universitat 1, 43204 Reus, Spain

E-mail: francesc.llerena@urv.cat 


\section{Introduction}

A cooperative game with transferable utility (hereafter game) describes a situation in which a society or community can profit from joint efforts. It consists of a finite set of players and a real-valued function defined on the set of coalitions of players. Assuming that the grand coalition will form, the question is how to allocate the gains from cooperation among the players. A single-valued solution (or rule) is a mapping that assigns to each game a feasible payoff vector, being one of the objectives of the axiomatic method to identify a solution by a set of appealing properties.

Probably, the most relevant single-valued solution is the Shapley value (Shapley, 1953b) which considers that players should be paid only according to their marginal contributions to all coalitions. In front of this marginality principle, the equal surplus division solution (Driessen and Funaki, 1991) ${ }^{1}$ relies on egalitarian considerations: it assigns to every player what they can achieve for themselves alone, and distributes equally what is left of the gains of cooperation. Both solutions satisfy equal treatment of equals. This property states that if two players have equal contributions to all coalitions, they must receive the same payoff. Nevertheless, in many applications, and because of external features of the players, the assumption that every player has the same abilities may not be appropriated. The weighted Shapley values (Shapley, 1953a) and the weighted surplus division solutions (Calleja and Llerena, 2016) take care of this aspect by assigning exogenously each player to a strictly positive weight, representing such abilities. A different prominent rule is the prenucleolus (Schmeidler, 1969) that takes specially care of minimizing complaints of coalitions to a particular allocation.

In this paper, we consider the problem of axiomatizing the weighted surplus division solution on the domain of all games. Despite the equal surplus division solution has recently been characterized, ${ }^{2}$ as far as we know, there is no proper axiomatic characterization of its non-symmetric generalization. Interestingly, our results show that, although the definitions of the weighted surplus division solutions, the weighted Shapley values and the prenucleolus differ completely, from an axiomatic approach the difference can be pointed out to one axiom: consistency, an outstanding relational property in the axiomatic method. ${ }^{3}$

Together with projection consistency (Funaki, 1998), Theorem 1 (i) characterizes the family of the weighted surplus division solutions by means of the well established property of covariance and path monotonicity, a sort of aggregate monotonicity (Megiddo, 1974) that distributes any variation in the worth of the grand coalition following a fixed pattern reflecting some exogenous circumstances or priorities among players that are not captured by the characteristic function of the game. From a different angle, and under efficiency, path monotonicity can be

1 This solution is also known as the center-of-gravity of the imputation set.

2 See, for instance, van den Brink (2007), Chun and Park (2012), Casajus and Huettner (2014), Béal at al. (2015), Calleja and Llerena (2016) or van den Brink et al. (2016).

3 See Thomson (2011) and Thomson (2012) for essays on the consistency principle. 
viewed as a relaxation of two equivalent properties (on the full domain of games): fairness, due to van den Brink (2001) and inspired in the notion of fairness as introduced in Myerson (1977), and differential marginality (Casajus, 2011). Fairness (marginality) guarantees that if we add a game to another game in which two players are symmetric then, since their marginal contributions to coalitions containing neither of them coincide in both games, the players' payoffs change by the same amount. Not surprisingly, fairness, together with the standard property of efficiency, imply path monotonicity. Outstandingly, replacing projection consistency in Theorem 1 (i) by either self consistency (Hart and Mas-Colell, 1989) or by max consistency (Davis and Maschler, 1965) and considering path monotonicity for two-person games only, we obtain new axiomatic characterizations of the family of weighted Shapley values (Theorem 1 (ii)) and the prenucleolus (Theorem 2). All these characterization results are collected in Section 3.

In Section 4 we observe that substituting path monotonicity in Theorem 1 by fairness allows to get new axiomatic characterizations of the equal surplus division solution (Theorem 3 (i)) and the Shapley value (Theorem 3 (ii)). Furthermore, the notion of duality in coalitional games (see, for instance, Oishi et al., 2016) leads to a new axiomatic characterization of the egalitarian non-separable contribution solution (Moulin, 1985) (Theorem 3 (iii)). ${ }^{4}$ Finally, replacing path monotonicity by fairness (for two-person games) in Theorem 2, we obtain a new characterization of the prenucleolus (Theorem 4).

The remainder of the paper is organized as follows. In Section 2 we introduce some preliminaries on games. In Section 5 we introduce the dual property of path monotonicity in order to provide, together with complement consistency (Moulin, 1985) and covariance, an axiomatic characterization of the family of weighted egalitarian non-separable contribution solutions (Theorem 7). We conclude with some remarks for future research and comparing our results with the characterizations provided in Sobolev (1975), Hart and Mas-Colell (1989), Orshan (1993) and Driessen and Funaki (1997). The Appendix contains the independence of the properties in the characterization results.

\section{Preliminaries}

The set of natural numbers $\mathbb{N}$ denotes the universe of potential players. A coalition is a non-empty finite subset of $\mathbb{N}$ and let $\mathcal{N}$ denote the set of all coalitions of $\mathbb{N}$. Given $S, T \in \mathcal{N}$, we use $S \subset T$ to indicate strict inclusion, that is, $S \subseteq T$ and $S \neq T$. By $|S|$ we denote the cardinality of the coalition $S \in \mathcal{N}$. A transferable utility coalitional game is a pair $(N, v)$ where $N \in \mathcal{N}$ is the set of players and $v$ : $2^{N} \longrightarrow \mathbb{R}$ is the characteristic function that assigns to each coalition $S \subseteq N$ a real number $v(S)$, representing what $S$ can achieve by agreeing to cooperate, with the convention that $v(\emptyset)=0$. Given a game $(N, v)$, the dual game $\left(N, v^{d}\right)$ is defined

\footnotetext{
4 Previous axiomatic characterizations of the egalitarian non-separable contribution solution can be found in Moulin (1985), Driessen and Funaki (1997) and Hwang (2006).
} 
by setting for all $S \subseteq N, v^{d}(S)=v(N)-v(N \backslash S)$. For simplicity of notation, and if no confusion arises, we write $v(i), v(i j), \ldots$ instead of $v(\{i\}), v(\{i, j\}), \ldots$ Ву $\Gamma$ we denote the class of all games.

Given $N \in \mathcal{N}$ and $\emptyset \neq N^{\prime} \subseteq N$, the unanimity game $\left(N, u_{N^{\prime}}\right)$ associated to $N^{\prime}$ is defined as $u_{N^{\prime}}(S)=1$ if $N^{\prime} \subseteq S$ and $u_{N^{\prime}}(S)=0$ otherwise. Given a game $(N, v)$ and $\emptyset \neq N^{\prime} \subset N$, the subgame $\left(N^{\prime}, v_{\mid N^{\prime}}\right)$ is defined as $v_{\mid N^{\prime}}(S)=v(S)$ for all $S \subseteq N^{\prime}$. For any two games $(N, v),(N, w), \alpha \in \mathbb{R}$ and $d \in \mathbb{R}^{N}$, we define the game $(N, v+w)$ as $(v+w)(S)=v(S)+w(S)$, the game $(N, \alpha \cdot v)$ as $(\alpha \cdot v)(S)=$ $\alpha \cdot v(S)$ and the game $(N, v+d)$ as $(v+d)(S)=v(S)+\sum_{i \in S} d_{i}$, for all $S \subseteq N$. The null game $(N, \mathbf{0})$ is defined by $\mathbf{0}(S)=0$ for all $S \subseteq N$.

Given $N \in \mathcal{N}$, let $\mathbb{R}^{N}$ stand for the space of real-valued vectors indexed by $N$, $x=\left(x_{i}\right)_{i \in N}$, and for all $S \subseteq N, x(S)=\sum_{i \in S} x_{i}$, with the convention $x(\emptyset)=0$. For each $x \in \mathbb{R}^{N}$ and $T \subseteq N, x_{\mid T}$ denotes the restriction of $x$ to $T: x_{\mid T}=\left(x_{i}\right)_{i \in T} \in \mathbb{R}^{T}$. Given two vectors $x, y \in \mathbb{R}^{N}, x \geq y$ if $x_{i} \geq y_{i}$, for all $i \in N$, while $x>y$ if $x_{i}>y_{i}$, for all $i \in N$.

The set of feasible payoff vectors of $(N, v)$ is defined by $X^{*}(N, v):=\{x \in$ $\left.\mathbb{R}^{N} \mid x(N) \leq v(N)\right\}$, while the preimputation set contains the efficient payoff vectors, that is, $X(N, v):=\left\{x \in \mathbb{R}^{N} \mid x(N)=v(N)\right\}$.

A solution on a class of games $\Gamma^{\prime} \subseteq \Gamma$ is a correspondence $\sigma$ that associates with each game $(N, v) \in \Gamma^{\prime}$ a subset $\sigma(N, v)$ of $X^{*}(N, v)$. Given a solution $\sigma$ on $\Gamma^{\prime} \subseteq \Gamma$ such that $(N, v),\left(N, v^{d}\right) \in \Gamma^{\prime}$, the dual of $\sigma$, denoted by $\sigma^{d}$, is defined by setting for all $(N, v) \in \Gamma^{\prime}, \sigma^{d}(N, v)=\sigma\left(N, v^{d}\right)$. A solution $\sigma$ on $\Gamma^{\prime} \subseteq \Gamma$ is said to be single-valued if $|\sigma(N, v)|=1$ for all $(N, v) \in \Gamma^{\prime}$. In this case, $\sigma(N, v)$ is treated as the unique element of this singleton set. Notice that a singlevalued solution is always non-empty but not necessarily an efficient allocation. We say that a single-valued solution $\sigma$ on $\Gamma^{\prime} \subseteq \Gamma$ satisfies efficiency (E) if all the gains from cooperation are shared among the players, that is, for all $N \in \mathcal{N}$ and all $(N, v) \in \Gamma^{\prime}$, it holds $\sum_{i \in N} \sigma_{i}(N, v)=v(N)$. Apart from efficiency, a classical invariant requirement w.r.t. changes in scale that are comparable with positive affine transformations is covariance. A single-valued solution $\sigma$ on $\Gamma^{\prime} \subseteq \Gamma$ satisfies covariance $(\mathbf{C O})$ if for all $N \in \mathcal{N}$, all $(N, v) \in \Gamma^{\prime}$, all $\alpha>0$ and all $d \in \mathbb{R}^{N}$, if $(N, \alpha \cdot v+d) \in \Gamma^{\prime}$ then $\sigma(N, \alpha \cdot v+d)=\alpha \cdot \sigma(N, v)+d$. Two players $i$ and $j$ are symmetric in a game $(N, v)$ if $v(S \cup\{i\})=v(S \cup\{j\})$ for all $S \subseteq N \backslash\{i, j\}$. A single-valued solution $\sigma$ on $\Gamma^{\prime} \subseteq \Gamma$ satisfies equal treatment of equals (ETE) if for all $N \in \mathcal{N}$, all $(N, v) \in \Gamma^{\prime}$ and all symmetric players $i, j \in N$, then $\sigma_{i}(N, v)=\sigma_{j}(N, v)$.

On the dual of games $\Gamma^{\prime} \subseteq \Gamma$ that is closed under the duality operator, two properties are dual to each other if whenever a solution $\sigma$ satisfies one of them, the dual solution $\sigma^{d}$ satisfies the other. A property is self dual if it is dual to itself. It is not difficult to check that efficiency, covariance and equal treatment of equals are self dual.

For our purposes, we introduce some well-known efficient single-valued solutions defined on $\Gamma$. Let $N \in \mathcal{N}$ and $(N, v) \in \Gamma$. The Shapley value, $S h$, is defined 
by

$$
S h_{i}(N, v):=\sum_{S \subseteq N \backslash\{i\}} \frac{|S| !(|N|-|S|-1) !}{|N| !}(v(S \cup\{i\})-v(S)) \text { for all } i \in N .
$$

Let $\alpha_{T}=\sum_{S \subseteq T}(-1)^{|T|-|S|} v(S)$ for all $\emptyset \neq T \subseteq N$. Then, we can express the game $(N, v)$ by a linear combination of the unanimity games as $v=\sum_{\emptyset \neq T \subseteq N} \alpha_{T} u_{T}$. The weighted Shapley value relative to a list of positive weights $w=\left(w_{i}\right)_{i \in \mathbb{N}} \in \mathbb{R}_{++}^{\mathbb{N}}$, $S h^{w}$, is defined by

$$
S h^{w}(N, v):=\sum_{\emptyset \neq T \subseteq N} \alpha_{T} \cdot S h^{w}\left(N, u_{T}\right)
$$

where

$$
S h_{i}^{w}\left(N, u_{T}\right):=\left\{\begin{array}{cc}
\frac{w_{i}}{\sum_{j \in T} w_{j}} & \text { if } i \in T \\
0 & \text { if } i \in N \backslash T
\end{array} .\right.
$$

Notice that when $w_{i}=w_{j}$ for all $i, j \in \mathbb{N}$, then $\operatorname{Sh}^{w}(N, v)=S h(N, v)$.

Let $N \in \mathcal{N}$ and $(N, v) \in \Gamma$. The equal surplus division solution, $E S$, is defined by

$$
E S_{i}(N, v):=v(i)+\frac{1}{|N|}\left(v(N)-\sum_{j \in N} v(j)\right) \text { for all } i \in N .
$$

The weighted surplus division solution relative to a list of positive weights $w=\left(w_{i}\right)_{i \in \mathbb{N}} \in \mathbb{R}_{++}^{\mathbb{N}}, E S^{w}$, is defined by

$$
E S_{i}^{w}(N, v):=v(i)+\frac{w_{i}}{\sum_{j \in N} w_{j}}\left(v(N)-\sum_{j \in N} v(j)\right) \text { for all } i \in N .
$$

Given a list of positive weights $w, E S^{w}$ can be interpreted as a two-stage rule: after assigning to every player what they can achieve for themselves alone, it distributes what is left of the gains of cooperation proportionally according to $w$, representing some exogenous abilities or bargaining power of the players. Notice that when $w_{i}=w_{j}$ for all $i, j \in \mathbb{N}$, then $E S^{w}(N, v)=E S(N, v)$.

The dual solutions of $E S$ and $E S^{w}$ are, respectively, the egalitarian nonseparable contribution solution, $E N S C$, and the weighted egalitarian nonseparable contribution solution, $E N S C^{w}$. Let $N \in \mathcal{N}$ and $(N, v) \in \Gamma$. The $E N S C$ is defined by setting, for all $i \in N$,

$$
\operatorname{ENSC}_{i}(N, v):=M_{i}(v)+\frac{1}{|N|}\left(v(N)-\sum_{j \in N} M_{j}(v)\right)
$$

where $M_{j}(v)=v(N)-v(N \backslash\{j\})$, for all $j \in N$. The $E N S C^{w}$ relative a list of positive weights $w=\left(w_{i}\right)_{i \in \mathbb{N}} \in \mathbb{R}_{++}^{\mathbb{N}}$ is given, for all $i \in N$, by

$$
\operatorname{ENSC}_{i}^{w}(N, v):=M_{i}(v)+\frac{w_{i}}{\sum_{j \in N} w_{j}}\left(v(N)-\sum_{j \in N} M_{j}(v)\right) .
$$


Let $N \in \mathcal{N}$ and $(N, v) \in \Gamma$. With any preimputation $x \in X(N, v)$ we associate the vector of all excesses $e(S, x)=v(S)-x(S), \emptyset \neq S \subset N$, the components of which are non-increasingly ordered. The prenucleolus, $\nu_{*}$, is the preimputation that minimizes with respect to the lexicographic order ${ }^{5}$ the vector of excesses over the set of preimputations.

For the two-agent case, the weighted standard solution relative to a list of positive weights $w=\left(w_{i}\right)_{i \in \mathbb{N}} \in \mathbb{R}_{++}^{\mathbb{N}}, S T^{w}$, is defined as follows: for all $N=$ $\{i, j\} \in \mathcal{N}$ and all $(N, v) \in \Gamma$,

$$
\begin{aligned}
& S T_{i}^{w}(N, v):=v(i)+\frac{w_{i}}{w_{i}+w_{j}}(v(N)-v(i)-v(j)), \\
& S T_{j}^{w}(N, v):=v(j)+\frac{w_{j}}{w_{i}+w_{j}}(v(N)-v(i)-v(j)) .
\end{aligned}
$$

Many solutions in the literature coincide with the standard solution that show up when all the players have the same weight.

Given a list of positive weights $w \in \mathbb{R}_{++}^{\mathbb{N}}$, we say that a single-valued solution $\sigma$ on $\Gamma^{\prime} \subseteq \Gamma$ satisfies $w$-proportionality $(w-\mathbf{P})$ if for all $N=\{i, j\} \in \mathcal{N}$ and all $(N, v) \in \Gamma^{\prime}$, it holds $\sigma(N, v)=S T^{w}(N, v)$. If all weights are identical, we say that $\sigma$ satisfies standardness $(\mathbf{S T})$.

\section{Consistency and path monotonicity}

The main concern of this section is to characterize the weighted surplus division solution by means of consistency together with monotonicity and covariance. Interestingly, our characterization result shows that, from an axiomatic point of view, the consistency principle distinguishes the weighted surplus division solution, the weighted Shapley value and the prenucleolus.

Consistency is a sort of internal stability requirement that relates the solution of a game to the solution of a reduced game that results when some agents leave. The different ways in which the agents that remain evaluate the possible coalitions give rise to different notions of reduced game. Here we deal with four ways of reducing a game: the self reduced game (Hart and Mas-Colell, 1989), the projection reduced game (Funaki, 1998), the complement reduced game (Moulin, 1985) and the max reduced game (Davis and Maschler, 1965). The terminology is taken from Thomson (2003).

Definition 1 Let $\sigma$ be a single-valued solution, $N \in \mathcal{N},(N, v) \in \Gamma$, and $\emptyset \neq$ $N^{\prime} \subset N$. The self reduced game relative to $N^{\prime}$ at $\sigma$ is the game $\left(N^{\prime}, r_{S, \sigma}^{N^{\prime}}(v)\right)$ defined by

$r_{S, \sigma}^{N^{\prime}}(v)(R):= \begin{cases}0 & \text { if } R=\emptyset, \\ v\left(R \cup N \backslash N^{\prime}\right)-\sum_{i \in N \backslash N^{\prime}} \sigma_{i}\left(R \cup N \backslash N^{\prime}, v_{\mid R \cup N \backslash N^{\prime}}\right) & \text { if } \emptyset \neq R \subseteq N^{\prime} .\end{cases}$

\footnotetext{
${ }^{5}$ Given two vectors $x, y \in \mathbb{R}^{N}$, we say that $x \leq_{\text {lex }} y$ if either $x=y$, or $x_{1}<y_{1}$ or there exists $k \in\{2, \ldots,|N|\}$ such that $x_{i}=y_{i}$ for all $1 \leq i \leq k-1$ and $x_{k}<y_{k}$.
} 
Definition 2 Let $N \in \mathcal{N},(N, v) \in \Gamma, x \in \mathbb{R}^{N}$ and $\emptyset \neq N^{\prime} \subset N$. The projection reduced game relative to $N^{\prime}$ at $x$ is the game $\left(N^{\prime}, r_{P, x}^{N^{\prime}}(v)\right)$ defined by

$$
r_{P, x}^{N^{\prime}}(v)(R):= \begin{cases}v(R) & \text { if } R \subset N^{\prime} \\ v(N)-x\left(N \backslash N^{\prime}\right) & \text { if } R=N^{\prime}\end{cases}
$$

Definition 3 Let $N \in \mathcal{N},(N, v) \in \Gamma, x \in \mathbb{R}^{N}$ and $\emptyset \neq N^{\prime} \subset N$. The complement reduced game relative to $N^{\prime}$ at $x$ is the game $\left(N^{\prime}, r_{C, x}^{N^{\prime}}(v)\right)$ defined by

$$
r_{C, x}^{N^{\prime}}(v)(R):= \begin{cases}0 & \text { if } R=\emptyset, \\ v\left(R \cup N \backslash N^{\prime}\right)-x\left(N \backslash N^{\prime}\right) & \text { if } \emptyset \neq R \subseteq N^{\prime} .\end{cases}
$$

Definition 4 Let $N \in \mathcal{N},(N, v) \in \Gamma, x \in \mathbb{R}^{N}$ and $\emptyset \neq N^{\prime} \subset N$. The max reduced game relative to $N^{\prime}$ at $x$ is the game $\left(N^{\prime}, r_{M, x}^{N^{\prime}}(v)\right)$ defined by

$$
r_{M, x}^{N^{\prime}}(v)(R):= \begin{cases}0 & \text { if } R=\emptyset, \\ \max _{Q \subseteq N \backslash N^{\prime}}\{v(R \cup Q)-x(Q)\} & \text { if } \emptyset \neq R \subset N^{\prime}, \\ v(N)-x\left(N \backslash N^{\prime}\right) & \text { if } R=N^{\prime} .\end{cases}
$$

In the self reduced game (relative to $N^{\prime}$ at $\sigma$ ), the worth of a coalition $R \subseteq N^{\prime}$ is determined under the assumption that $R$ joins all members of $N \backslash N^{\prime}$, provided they are paid according to $\sigma$ in the subgame associated to $R \cup\left(N \backslash N^{\prime}\right)$. In the projection reduced game (relative to $N^{\prime}$ at $x$ ), when players in $N \backslash N^{\prime}$ leave the game, for a proper subcoalition $R \subset N^{\prime}$ cooperation is no longer possible with them. By contrast, in the complement reduced game (relative to $N^{\prime}$ at $x$ ) each coalition $R \subseteq N^{\prime}$ is required to join all the members of $N \backslash N^{\prime}$, provided that they are paid according to $x$. Finally, in the max reduced game (relative to $N^{\prime}$ at $x$ ), the worth of a coalition $R \subset N^{\prime}$ is determined under the assumption that $R$ can choose the best partners in $N \backslash N^{\prime}$, provided that they are paid according to $x$.

The following notions of consistency rely on the above definitions of reduced game.

A single-valued solution $\sigma$ on $\Gamma^{\prime} \subseteq \Gamma$ satisfies

- Self consistency (SC): if for all $N \in \mathcal{N}$, all $(N, v) \in \Gamma^{\prime}$, all $\emptyset \neq N^{\prime} \subset N$ and $x=\sigma(N, v)$, then $\left(N^{\prime}, r_{S, \sigma}^{N^{\prime}}(v)\right) \in \Gamma^{\prime}$ and $x_{\mid N^{\prime}}=\sigma\left(N^{\prime}, r_{S, \sigma}^{N^{\prime}}(v)\right)$.

- Projection consistency (PC): if for all $N \in \mathcal{N}$, all $(N, v) \in \Gamma^{\prime}$, all $\emptyset \neq N^{\prime} \subset$ $N$ and $x=\sigma(N, v)$, then $\left(N^{\prime}, r_{P, x}^{N^{\prime}}(v)\right) \in \Gamma^{\prime}$ and $x_{\mid N^{\prime}}=\sigma\left(N^{\prime}, r_{P, x}^{N^{\prime}}(v)\right)$.

- Complement consistency (CC): if for all $N \in \mathcal{N}$, all $(N, v) \in \Gamma^{\prime}$, all $\emptyset \neq$ $N^{\prime} \subset N$ and $x=\sigma(N, v)$, then $\left(N^{\prime}, r_{C, x}^{N^{\prime}}(v)\right) \in \Gamma^{\prime}$ and $x_{\mid N^{\prime}}=\sigma\left(N^{\prime}, r_{C, x}^{N^{\prime}}(v)\right)$.

- Max consistency (MC): if for all $N \in \mathcal{N}$, all $(N, v) \in \Gamma^{\prime}$, all $\emptyset \neq N^{\prime} \subset N$, and $x=\sigma(N, v)$, then $\left(N^{\prime}, r_{M, x}^{N^{\prime}}(v)\right) \in \Gamma^{\prime}$ and $x_{\mid N^{\prime}}=\sigma\left(N^{\prime}, r_{M, x}^{N^{\prime}}(v)\right)$.

The consistency principle states that in the corresponding reduced game the original agreement should be confirmed. Funaki (1998) shows that projection consistency and complement consistency are dual properties. 
The next result links consistency with covariance (for two-person games) and efficiency.

Proposition 1 Let $\sigma$ be a single-valued solution on $\Gamma$ that satisfies either projection consistency, complement consistency, self consistency or max consistency and, for two-person games, covariance. Then, $\sigma$ satisfies efficiency.

Proof Let $\sigma$ be a single-valued solution on $\Gamma$ that satisfies $\mathbf{C O}$ for two-person games and PC. Let $(\{i, j\}, \mathbf{0})$ be the null game. Then, by $\mathbf{C O}$ (for two-person games) we have $\sigma(\{i, j\}, \mathbf{0})=\sigma(\{i, j\}, 2 \cdot \mathbf{0})=2 \cdot \sigma(\{i, j\}, \mathbf{0})$ and, consequently, $\sigma(\{i, j\}, \mathbf{0})=(0,0)$. Let $(\{i, j\}, v)$ be a game such that $v(i j)=v(i)+v(j)$. Then, by $\mathbf{C O}$ (for two-person games) we have

$\sigma(\{i, j\}, v)=\sigma(\{i, j\}, 1 \cdot \mathbf{0}+(v(i), v(j))=1 \cdot \sigma(\{i, j\}, \mathbf{0})+(v(i), v(j))=(v(i), v(j))$.

Now, let $(\{i\}, v)$ be a one-person game and, for some $j \in \mathbb{N} \backslash\{i\}$, consider the game $\left(\{i, j\}, v^{\prime}\right)$ defined by $v^{\prime}(i)=v^{\prime}(i j)=v(i)$ and $v^{\prime}(j)=0$. Since $v^{\prime}(i j)=$ $v^{\prime}(i)+v^{\prime}(j)$, from (1) it comes that $\sigma_{i}\left(\{i, j\}, v^{\prime}\right)=v(i)$ and $\sigma_{j}\left(\{i, j\}, v^{\prime}\right)=0$. It is easy to check that $(\{i\}, v)=\left(\{i\}, r_{P, x}^{\{i\}}\left(v^{\prime}\right)\right)$ being $x=\sigma\left(\{i, j\}, v^{\prime}\right)$. By PC, $\sigma(\{i\}, v)=v(i)$ which implies efficiency for one-person games. Let $N \in \mathcal{N}$ with $|N| \geq 2,(N, v) \in \Gamma$ and $i \in N$. Then, efficiency for one-person games implies $\sigma_{i}\left(\{i\}, r_{P, x}^{\{i\}}(v)\right)=r_{P, x}^{\{i\}}(v)(i)=v(N)-\sum_{j \in N \backslash\{i\}} \sigma_{j}(N, v)$, where $x=\sigma(N, v)$. By $\mathbf{P C}, \sigma_{i}(N, v)=\sigma_{i}\left(\{i\}, r_{P, x}^{\{i\}}(v)\right)$ and thus $\sigma_{i}(N, v)=v(N)-\sum_{j \in N \backslash\{i\}} \sigma_{j}(N, v)$, which proves $\mathbf{E}$.

The same arguments hold replacing $\mathbf{P C}$ by either $\mathbf{C C}, \mathbf{S C}$ or $\mathbf{M C}$.

Several notions of monotonicity have played a role in characterizing solutions on different frameworks. ${ }^{6}$ In this section, we introduce a variant of aggregate monotonicity (Megiddo, 1974), which states that nobody is worse off whenever the worth of the grand coalition increases, while the worth of every other coalition remains unchanged. By imposing some regularity in the way players share the extra profits (loses) if only the worth of the grand coalition increases (decreases), we introduce path monotonicity.

Definition 5 A monotone path is a function $f: \mathcal{N} \times \mathbb{R} \rightarrow \bigcup_{N \in \mathcal{N}} \mathbb{R}^{N}$ satisfying the following conditions: for all $N \in \mathcal{N}$ and all $t \in \mathbb{R}$,

1. $f(N, 0)=(0, \ldots, 0) \in \mathbb{R}^{N}$,

2. $f(N, t) \in \mathbb{R}^{N}$ and $\sum_{i \in N} f_{i}(N, t)=t$,

3. if $t^{\prime} \in \mathbb{R}$ is such that $t^{\prime}>t$, then $f_{i}\left(N, t^{\prime}\right)>f_{i}(N, t)$ for all $i \in N$.

Notice that a monotone path assigns positive (negative) vectors to positive (negative) real numbers. Let $\mathcal{F}_{\text {mon }}$ denote the class of monotone paths.

A monotone path $f$ specifies a complete list of monotonic agreements. We assume that whenever a set of players $N \in \mathcal{N}$ reaches an agreement (which can be

\footnotetext{
${ }^{6}$ See, for instance, Kalai and Smorodinsky (1975), Kalai (1977), Kalai and Samet (1985), Young (1985) or Thomson (1987).
} 
different for different sets) on how to distribute monotonically an amount $t \in \mathbb{R}$, representing the difference of the worth of the grand coalitions between two games, they will respect this agreement by following the same principle, regardless of the games they eventually play.

The family $\mathcal{F}_{\text {mon }}$ is very rich. Possibly, the simplest monotone path is to consider that all players should be treated equally: for all $N \in \mathcal{N}$, all $t \in \mathbb{R}$ and all $i \in N$,

$$
\bar{f}_{i}(N, t)=\frac{t}{|N|} .
$$

However, path monotonicity allows for treating players differently to reflect some individual abilities or exogenous circumstances among them (like income or health status) that are not captured by the mathematical description of the game. For instance, given a list of exogenous weights $w \in \mathbb{R}_{++}^{\mathbb{N}}$, define, for all $N \in \mathcal{N}$, all $t \in \mathbb{R}$ and all $i \in N$,

$$
f_{i}^{w}(N, t)=\frac{w_{i}}{\sum_{j \in N} w_{j}} \cdot t
$$

A single-valued solution $\sigma$ on $\Gamma^{\prime} \subseteq \Gamma$ satisfies

- Path monotonicity (P-MO): if there exists a monotone path $f \in \mathcal{F}_{\text {mon }}$ such that, for all $N \in \mathcal{N}$ and all $(N, v),\left(N, v^{\prime}\right) \in \Gamma^{\prime}$ with $v(S)=v^{\prime}(S)$ for all $S \subset N$, it holds $\sigma\left(N, v^{\prime}\right)-\sigma(N, v)=f\left(N, v^{\prime}(N)-v(N)\right)$.

Obviously, path monotonicity implies aggregate monotonicity, but the next example shows that the reverse implication is not true.

Example 1 Define the single-valued solution $\rho$ by setting, for all $N \in \mathcal{N}$, all $(N, v) \in \Gamma$ and all $i \in N$,

$$
\rho_{i}(v):=v(i)+\frac{|\{j \in N \mid v(i) \geq v(j)\}|}{\sum_{k \in N}|\{j \in N \mid v(k) \geq v(j)\}|} \cdot\left(v(N)-\sum_{j \in N} v(j)\right) .
$$

Clearly, $\rho$ satisfies aggregate monotonicity. Assume that $\rho$ also satisfies path monotonicity w.r.t. $f \in \mathcal{F}_{\text {mon }}$, and consider two games $\left(N, v^{1}\right)$ and $\left(N, v^{2}\right)$ with player set $N=\{1,2\}$ and characteristic functions: $v^{1}(1)=v^{2}(2)=1, v^{1}(2)=v^{2}(1)=0$ and $v^{1}(N)=v^{2}(N)=2$. Now define the associated games $\left(N,\left(v^{1}\right)^{\prime}\right)$ and $\left(N,\left(v^{2}\right)^{\prime}\right)$ by $\left(v^{1}\right)^{\prime}=v^{1}-u_{N}$ and $\left(v^{2}\right)^{\prime}=v^{2}-u_{N}$, respectively. Notice that, (a) $\rho_{1}\left(N, v^{1}\right)=$ $\frac{5}{3}, \rho_{1}\left(N,\left(v^{1}\right)^{\prime}\right)=1$ and (b) $\rho_{1}\left(N, v^{2}\right)=\frac{1}{3}, \rho_{1}\left(N,\left(v^{2}\right)^{\prime}\right)=0$. Then, from (a), $f_{1}(N, 1)=\frac{2}{3}$ and, from $(\mathrm{b}), f_{1}(N, 1)=\frac{1}{3}$, resulting in a contradiction. Hence, $\rho$ is not path monotonic.

The Shapley value, the equal surplus division solution and the egalitarian nonseparable contribution solution satisfy path monotonicity (w.r.t. $\bar{f}$ ). For any list of positive weights $w \in \mathbb{R}_{++}^{\mathbb{N}}$, the weighted Shapley value and the weighted surplus division solution also meet path monotonicity (w.r.t. $f^{w}$ ). However, not all weighted egalitarian non-separable contribution solution satisfies aggregate monotonicity, and so path monotonicity. Let us provide an example to check this point. 
Example 2 Consider two games $(N, v)$ and $\left(N, v^{\prime}\right)$ with set of players $N=\{1,2,3\}$ and characteristic functions as follow: $v(S)=v^{\prime}(S)$ for all $S \subset N, v(i)=0$ for all $i \in N, v(12)=v(13)=1, v(23)=0, v(N)=1$ and $v^{\prime}(N)=2$. Let $w \in \mathbb{R}_{++}^{\mathbb{N}}$ be a list of positive weights such that $w_{1}=3$ and $w_{2}=w_{3}=1$. A routine calculus shows that $\operatorname{ENSC}^{w}(v)=(1,0,0)$ and $E N S C^{w}\left(v^{\prime}\right)=\left(\frac{4}{5}, \frac{3}{5}, \frac{3}{5}\right)$. Hence, ENSC $C^{w}$ is not aggregate monotonic (and neither path monotonic).

Notice that, since $E S^{w}$ and $E N S C^{w}$ are dual to each other and $E S^{w}$ satisfies path monotonicity, from Example 2 we may conclude that path monotonicity is not self dual.

The next remark highlights that path monotonicity yields some structure on the associated monotonic function $f \in \mathcal{F}_{\text {mon }}$.

Remark 1 Interestingly, if $\sigma$ is a single-valued solution on $\Gamma$ satisfying path monotonicity w.r.t $f \in \mathcal{F}_{\text {mon }}$, then $f$ is additive. That is, for all $N \in \mathcal{N}$ and all $t, t^{\prime} \in \mathbb{R}$, $f\left(N, t+t^{\prime}\right)=f(N, t)+f\left(N, t^{\prime}\right)$. To show it, consider three games $(N, v),\left(N, v^{\prime}\right)$ and $\left(N, v^{\prime \prime}\right)$ such that $v(S)=v^{\prime}(S)=v^{\prime \prime}(S)$ for all $S \subset N, v(N)-v^{\prime}(N)=t$ and $v^{\prime}(N)-v^{\prime \prime}(N)=t^{\prime}$. By path monotonicity, we have

$$
\begin{aligned}
f\left(N, t+t^{\prime}\right) & =f\left(N, v(N)-v^{\prime \prime}(N)\right)=\sigma(N, v)-\sigma\left(N, v^{\prime \prime}\right) \\
& =\sigma(N, v)-\sigma\left(N, v^{\prime}\right)+\sigma\left(N, v^{\prime}\right)-\sigma\left(N, v^{\prime \prime}\right) \\
& =f\left(N, v(N)-v^{\prime}(N)\right)+f\left(N, v^{\prime}(N)-v^{\prime \prime}(N)\right) \\
& =f(N, t)+f\left(N, t^{\prime}\right) .
\end{aligned}
$$

Moreover, if an additive function $g: \mathbb{R} \rightarrow \mathbb{R}$ is monotonic, then there exists a constant $k \in \mathbb{R}$ such that $g(x)=k \cdot x$ for all $x \in \mathbb{R}$ (see, for instance, Theorem 2.1 in Jung, 2011). Hence, there exist $k_{1}, \ldots, k_{n} \in \mathbb{R}$ such that, for all $N \in \mathcal{N}$ and all $\alpha, t \in \mathbb{R}, f(N, \alpha \cdot t)=\left(k_{1} \cdot \alpha \cdot t, \ldots, k_{n} \cdot \alpha \cdot t\right)=\alpha \cdot\left(k_{1} \cdot t, \ldots, k_{n} \cdot t\right)=\alpha \cdot f(N, t)$. Consequently, $f \in \mathcal{F}_{\text {mon }}$ is homogeneous.

The next two lemmas are important to prove an intermediate result showing that imposing, for two-person games, covariance and path monotonicity, consistency enables us to generate endogenously a collection of positive weights.

Lemma 1 Let $\sigma$ be a single-valued solution on $\Gamma$ that satisfies, for two-person games, covariance and path monotonicity. Then, for all $\{i, j\} \in \mathcal{N}$ it holds

(i) $\sigma\left(\{i, j\}, u_{\{i, j\}}\right)>(0,0)$.

(ii) $\sigma\left(\{i, j\}, u_{\{i, j\}}\right)=-\sigma\left(\{i, j\},-u_{\{i, j\}}\right)$.

Proof Let $\sigma$ be a single-valued solution on $\Gamma$ that satisfies $\mathbf{C O}$ and P-MO for twoperson games. Let $N=\{i, j\}$ and consider the associated unanimity game $\left(N, u_{N}\right)$. By P-MO (for two-person games), there exists a monotone path $f \in \mathcal{F}_{\text {mon }}$ such that $\sigma\left(N, u_{N}\right)=\sigma(N, \mathbf{0})+f(N, 1)$. Similarly, $\sigma\left(N,-u_{N}\right)=\sigma(N, \mathbf{0})+f(N,-1)$. By CO (for two-person games), $\sigma(N, \mathbf{0})=(0,0)$ (see expression (1) in Proposition 1 ) and thus $\sigma\left(N, u_{N}\right)=f(N, 1)$ and $\sigma\left(N,-u_{N}\right)=f(N,-1)$. Hence, $\sigma\left(N, u_{N}\right)>$ $(0,0)$ which proves $(i)$. Moreover, since $f$ is homogeneous (see Remark 1 ) we have $\sigma\left(N, u_{N}\right)+\sigma\left(N,-u_{N}\right)=f(N, 1)+f(N,-1)=(0,0)$, which proves $(i i)$. 
Lemma 2 Let $\sigma$ be a single-valued solution on $\Gamma$ that satisfies, for two-person games, covariance and path monotonicity. Let $N \in \mathcal{N}$ with $|N|=3$. Then, for all $k, s \in N$,

(i) if $\sigma$ satisfies either projection consistency or self consistency, it holds

$$
\frac{\sigma_{k}\left(N, u_{N}\right)}{\sigma_{s}\left(N, u_{N}\right)}=\frac{\sigma_{k}\left(\{k, s\}, u_{\{k, s\}}\right)}{\sigma_{s}\left(\{k, s\}, u_{\{k, s\}}\right)} .
$$

(ii) if $\sigma$ satisfies max consistency, it holds

$$
\frac{\sigma_{k}\left(N, u_{N}\right)-r_{M, x}^{\{k, s\}}\left(u_{N}\right)(k)}{\sigma_{s}\left(N, u_{N}\right)-r_{M, x}^{\{k, s\}}\left(u_{N}\right)(s)}=\frac{\sigma_{k}\left(\{k, s\}, u_{\{k, s\}}\right)}{\sigma_{s}\left(\{k, s\}, u_{\{k, s\}}\right)},
$$

where $x=\sigma\left(N, u_{N}\right)$.

Proof Let $\sigma$ be a single-valued solution on $\Gamma$ that satisfies, for two-person games, $\mathbf{C O}$ and P-MO.

(i) If $\sigma$ satisfies $\mathbf{P C}$, let $N \in \mathcal{N}$ with $|N|=3$ and denote $x=\sigma\left(N, u_{N}\right)$.

For all pairs of agents $k, s \in N$, by $\mathbf{P C}$ it holds that

$$
\sigma_{\mid\{k, s\}}\left(N, u_{N}\right)=\sigma\left(\{k, s\}, r_{P, x}^{\{k, s\}}\left(u_{N}\right)\right) .
$$

Let $\alpha=r_{P, x}^{\{k, s\}}\left(u_{N}\right)(k s)$. By the definition of projection reduced game,

$$
r_{P, x}^{\{k, s\}}\left(u_{N}\right)=\alpha \cdot u_{\{k, s\}} \text {. }
$$

If $\alpha>0$, by $\mathbf{C O}$ (for two-person games) it follows

$$
\sigma\left(\{k, s\}, r_{P, x}^{\{k, s\}}\left(u_{N}\right)\right)=\alpha \cdot \sigma\left(\{k, s\}, u_{\{k, s\}}\right) .
$$

If $\alpha=0$, as in the proof of Proposition 1 we have

$$
\sigma\left(\{k, s\}, r_{P, x}^{\{k, s\}}\left(u_{N}\right)\right)=(0,0) .
$$

If $\alpha<0$, notice first that $\alpha \cdot u_{\{k, s\}}=-\alpha \cdot\left(-u_{\{k, s\}}\right)$. Then,

$$
\begin{aligned}
\sigma\left(\{k, s\}, r_{P, x}^{\{k, s\}}\left(u_{N}\right)\right) & =\sigma\left(\{k, s\},-\alpha \cdot\left(-u_{\{k, s\}}\right)\right) \\
& =-\alpha \cdot \sigma\left(\{k, s\},-u_{\{k, s\}}\right) \\
& =\alpha \cdot \sigma\left(\{k, s\}, u_{\{k, s\}}\right),
\end{aligned}
$$

where the second equality holds by $\mathbf{C O}$ (for two-person games) and the last one by Lemma 1 (ii). Thus,

$$
\begin{aligned}
\sigma_{\mid\{k, s\}}\left(N, u_{N}\right) & =\sigma\left(\{k, s\}, r_{P, x}^{\{k, s\}}\left(u_{N}\right)\right) \\
& =\left(r_{P, x}^{\{k, s\}}\left(u_{N}\right)(k s)\right) \cdot \sigma\left(\{k, s\}, u_{\{k, s\}}\right) .
\end{aligned}
$$

By Lemma 1 (i), $\sigma\left(\{k, s\}, u_{\{k, s\}}\right)>(0,0)$, which implies that $\sigma_{k}\left(N, u_{N}\right)$ and $\sigma_{s}\left(N, u_{N}\right)$ have the same sign. By Proposition 1, $\sigma$ satisfies $\mathbf{E}$, then $\sum_{i \in N} \sigma_{i}\left(N, u_{N}\right)=1$ and thus $\sigma_{i}\left(N, u_{N}\right)>0$ for all $i \in N$. Finally, from (6) it follows (4).

The same arguments hold replacing $\mathbf{P C}$ by $\mathbf{S C}$. 
(ii) If $\sigma$ satisfies $\mathbf{M C}$, let $N \in \mathcal{N}$ with $|N|=3$ and denote $x=\sigma\left(N, u_{N}\right)$.

For all pairs of agents $k, s \in N$, by $\mathbf{M C}$ it holds that

$$
\sigma_{\mid\{k, s\}}\left(N, u_{N}\right)=\sigma\left(\{k, s\}, r_{M, x}^{\{k, s\}}\left(u_{N}\right)\right) .
$$

Let $\alpha=r_{M, x}^{\{k, s\}}\left(u_{N}\right)(k s)-r_{M, x}^{\{k, s\}}\left(u_{N}\right)(k)-r_{M, x}^{\{k, s\}}\left(u_{N}\right)(s)$. By the definition of max reduced game,

$$
r_{M, x}^{\{k, s\}}\left(u_{N}\right)=\alpha \cdot u_{\{k, s\}}+\left(r_{M, x}^{\{k, s\}}\left(u_{N}\right)(k), r_{M, x}^{\{k, s\}}\left(u_{N}\right)(s)\right) .
$$

If $\alpha>0$, by $\mathbf{C O}$ (for two-person games) it follows

$\sigma\left(\{k, s\}, r_{M, x}^{\{k, s\}}\left(u_{N}\right)\right)=\alpha \cdot \sigma\left(\{k, s\}, u_{\{k, s\}}\right)+\left(r_{M, x}^{\{k, s\}}\left(u_{N}\right)(k), r_{M, x}^{\{k, s\}}\left(u_{N}\right)(s)\right)$.

If $\alpha=0$, as in the proof of Proposition 1 we have

$$
\sigma\left(\{k, s\}, r_{M, x}^{\{k, s\}}\left(u_{N}\right)\right)=\left(r_{M, x}^{\{k, s\}}\left(u_{N}\right)(k), r_{M, x}^{\{k, s\}}\left(u_{N}\right)(s)\right) .
$$

If $\alpha<0$, notice first that $\alpha \cdot u_{\{k, s\}}=-\alpha \cdot\left(-u_{\{k, s\}}\right)$. Then,

$$
\begin{aligned}
\sigma\left(\{k, s\}, r_{M, x}^{\{k, s\}}\left(u_{N}\right)\right) & =\sigma\left(\{k, s\},-\alpha \cdot\left(-u_{\{k, s\}}\right)+\left(r_{M, x}^{\{k, s\}}\left(u_{N}\right)(k), r_{M, x}^{\{k, s\}}\left(u_{N}\right)(s)\right)\right) \\
& =-\alpha \cdot \sigma\left(\{k, s\},-u_{\{k, s\}}\right)+\left(r_{M, x}^{\{k, s\}}\left(u_{N}\right)(k), r_{M, x}^{\{k, s\}}\left(u_{N}\right)(s)\right) \\
& =\alpha \cdot \sigma\left(\{k, s\}, u_{\{k, s\}}\right)+\left(r_{M, x}^{\{k, s\}}\left(u_{N}\right)(k), r_{M, x}^{\{k, s\}}\left(u_{N}\right)(s)\right),
\end{aligned}
$$

where the second equality holds by CO (for two-person games) and the last one by Lemma 1 (ii). Thus,

$$
\begin{aligned}
& \sigma_{\mid\{k, s\}}\left(N, u_{N}\right)=\sigma\left(\{k, s\}, r_{M, x}^{\{k, s\}}\left(u_{N}\right)\right) \\
& =\left(r_{M, x}^{\{k, s\}}\left(u_{N}\right)(k s)-r_{M, x}^{\{k, s\}}\left(u_{N}\right)(k)-r_{M, x}^{\{k, s\}}\left(u_{N}\right)(s)\right) \cdot \sigma\left(\{k, s\}, u_{\{k, s\}}\right) \\
& +\left(r_{M, x}^{\{k, s\}}\left(u_{N}\right)(k), r_{M, x}^{\{k, s\}}\left(u_{N}\right)(s)\right) \text {. }
\end{aligned}
$$

By Lemma 1 (i), $\sigma\left(\{k, s\}, u_{\{k, s\}}\right)>(0,0)$, which implies

$$
\operatorname{Sign}\left(\sigma_{k}\left(N, u_{N}\right)-r_{M, x}^{\{k, s\}}\left(u_{N}\right)(k)\right)=\operatorname{Sign}\left(\sigma_{s}\left(N, u_{N}\right)-r_{M, x}^{\{k, s\}}\left(u_{N}\right)(s)\right) .
$$

We claim that

$$
\sigma_{k}\left(N, u_{N}\right)-r_{M, x}^{\{k, s\}}\left(u_{N}\right)(k) \neq 0 .
$$

Suppose, on the contrary, $\sigma_{k}\left(N, u_{N}\right)-r_{M, x}^{\{k, s\}}\left(u_{N}\right)(k)=0$. Then, by equality $(8), \sigma_{s}\left(N, u_{N}\right)-r_{M, x}^{\{k, s\}}\left(u_{N}\right)(s)=0$. Consequently,

$$
\begin{aligned}
& \sigma_{k}\left(N, u_{N}\right)=r_{M, x}^{\{k, s\}}\left(u_{N}\right)(k)=\max \left\{0,0-\sigma_{l}\left(N, u_{N}\right)\right\} \geq 0, \\
& \sigma_{s}\left(N, u_{N}\right)=r_{M, x}^{\{k, s\}}\left(u_{N}\right)(s)=\max \left\{0,0-\sigma_{l}\left(N, u_{N}\right)\right\} \geq 0,
\end{aligned}
$$

being $l \in N \backslash\{k, s\}$. Thus,

$$
\sigma_{k}\left(N, u_{N}\right)=\sigma_{s}\left(N, u_{N}\right) \geq 0 .
$$


Now consider the max reduced game $\left(\{k, l\}, r_{M, x}^{\{k, l\}}\left(u_{N}\right)\right)$ relative to $\{k, l\}$ at $x=\sigma\left(N, u_{N}\right)$. From (11) it follows that

$$
r_{M, x}^{\{k, l\}}\left(u_{N}\right)(k)=r_{M, x}^{\{k, l\}}\left(u_{N}\right)(l)=0 \text { and } r_{M, x}^{\{k, l\}}\left(u_{N}\right)(k l)=1-\sigma_{s}\left(N, u_{N}\right)
$$

By MC and, for two-person games, P-MO and CO (from which it follows expression (1) in the proof of Proposition 1), there is a monotone path $f \in$ $\mathcal{F}_{\text {mon }}$ such that

$$
\begin{aligned}
\sigma_{\mid\{k, l\}}\left(N, u_{N}\right) & =\sigma\left(\{k, l\}, r_{M, x}^{\{k, l\}}\left(u_{N}\right)\right) \\
& =\sigma(\{k, l\}, \mathbf{0})+f\left(\{k, l\}, 1-\sigma_{s}\left(N, u_{N}\right)\right) \\
& =f\left(\{k, l\}, 1-\sigma_{s}\left(N, u_{N}\right)\right) .
\end{aligned}
$$

Since $f \in \mathcal{F}_{\text {mon }}$ and, by $(11), \sigma_{k}\left(N, u_{N}\right) \geq 0$, we have $1-\sigma_{s}\left(N, u_{N}\right) \geq 0$. Consequently, $\sigma_{l}\left(N, u_{N}\right)=f_{l}\left(\{k, l\}, 1-\sigma_{s}\left(N, u_{N}\right)\right) \geq 0$. By expression (10), this means that $\sigma_{k}\left(N, u_{N}\right)=\sigma_{s}\left(N, u_{N}\right)=0$ and by $\mathbf{E}$ (see Proposition 1), $\sigma_{l}\left(N, u_{N}\right)=1$. Then, $\sigma_{l}\left(N, u_{N}\right)-r_{M, x}^{\{k, l\}}\left(u_{N}\right)(l)=1-0=1$. Since expression (8) holds for any pair of agents, we have $\sigma_{k}\left(N, u_{N}\right)-r_{M, x}^{\{k, l\}}\left(u_{N}\right)(k)>0$, in contradiction with $\sigma_{k}\left(N, u_{N}\right)=0$. This proves the claim, that is, inequality (9). But then, from (7) it follows (5).

Next, we connect consistency, covariance and path monotonicity with $w$-proportionality.

Proposition 2 Let $\sigma$ be a single-valued solution on $\Gamma$ that satisfies either projection consistency, self consistency or max consistency and, for two-person games, covariance and path monotonicity. Then, $\sigma$ satisfies $w$-proportionality.

Proof Let $\sigma$ be a single-valued solution on $\Gamma$ that satisfies $\mathbf{P C}$ and, for two-person games, $\mathbf{C O}$ and P-MO. By Proposition 1, $\sigma$ satisfies E. Next we see that $\sigma$ satisfies $w-\mathbf{P}$ w.r.t. the following collection of weights: fix a player $\mathbf{l} \in \mathbb{N}$ and define

$$
w_{k}=\left\{\begin{array}{cc}
1 & \text { if } k=\mathbf{l} \\
\frac{\sigma_{k}\left(\{k, \mathbf{l}\}, u_{\{k, \mathbf{l}\}}\right)}{\sigma_{\mathbf{l}}\left(\{k, \mathbf{l}\}, u_{\{k, \mathbf{l}\}}\right)} & \text { otherwise }
\end{array}\right.
$$

By Lemma 1 (i), $w$ is well defined.

Let $(N, v)$ be a game. If $N=\{i\}$, by $\mathbf{E}$ we have $\sigma(\{i\}, v)=v(i)+\frac{w_{i}}{w_{i}}(v(i)-$ $v(i))=E S^{w}(\{i\}, v)$. If $|N|=2$ we distinguish two cases:

1. Case 1: $N=\{1, i\}$.

Let us denote $\alpha=v(N)-v(\mathbf{l})-v(i)$.

If $\alpha=0$, then by $\mathbf{C O}$ (for two-person games) (see expression (1) in the proof of Proposition 1) we have $\sigma(N, v)=(v(\mathbf{l}), v(i))=S T^{w}(N, v)$. 
If $\alpha>0$, then for all $k \in N$, we have

$$
\begin{aligned}
\sigma_{k}(N, v) & =\sigma_{k}\left(N, \alpha \cdot u_{N}+(v(\mathbf{l}), v(i))\right) \\
& =\alpha \cdot \sigma_{k}\left(N, u_{N}\right)+v(k) \\
& =\alpha \cdot \frac{\sigma_{k}\left(N, u_{N}\right)}{\sigma_{\mathbf{l}}\left(N, u_{N}\right)+\sigma_{i}\left(N, u_{N}\right)}+v(k) \\
& =\alpha \cdot \frac{\frac{\sigma_{k}\left(N, u_{N}\right)}{\sigma_{1}\left(N, u_{N}\right)}}{1+\frac{\sigma_{i}\left(N, u_{N}\right)}{\sigma_{1}\left(N, u_{N}\right)}}+v(k) \\
& =\alpha \cdot \frac{w_{k}}{w_{\mathbf{l}}+w_{i}}+v(k) \\
& =S T_{k}^{w}(N, v)
\end{aligned}
$$

where the second and the third equalities follow by $\mathbf{C O}$ (for two-person games) and $\mathbf{E}$, respectively.

If $\alpha<0$, notice first that $v=-\alpha \cdot\left(-u_{N}\right)+(v(\mathbf{l}), v(i))$. By $\mathbf{C O}$ (for two-person games) and Lemma 1 (ii), $\sigma(N, v)=\alpha \cdot \sigma\left(N, u_{N}\right)+(v(\mathbf{l}), v(i))$. Now, following the reasoning above we obtain $\sigma_{k}(N, v)=S T_{k}^{w}(N, v)$, for all $k \in N$.

2. Case 2: $N=\{i, j\}$ and $\mathbf{l} \notin N$.

By the definition of $w$, Lemma 2 (i) and $\mathbf{E}$, it follows that

$$
\begin{aligned}
\frac{w_{i}}{w_{i}+w_{j}} & =\frac{1}{1+\frac{w_{j}}{w_{i}}} \\
& =\frac{1}{1+\frac{\sigma_{j}\left(\{j, 1\}, u_{\{j, 1\}}\right)}{\sigma_{1}\left(\{j, 1\}, u_{\{j, 1\}}\right)} \cdot \frac{\sigma_{\mathbf{1}}\left(\{i, \mathbf{1}\}, u_{\{i, 1\}}\right)}{\sigma_{i}\left(\{i, \mathbf{1}\}, u_{\{i, 1\}}\right)}} \\
& =\frac{1}{1+\frac{\sigma_{j}\left(\{i, j, 1\}, u_{\{i, j, 1\}}\right)}{\sigma_{\mathbf{1}}\left(\{i, j, 1\}, u_{\{i, j, 1\}}\right)} \cdot \frac{\sigma_{\mathbf{1}}\left(\{i, j, 1\}, u_{\{i, j, 1\}}\right)}{\sigma_{i}\left(\{i, j, 1\}, u_{\{i, j, 1\}}\right)}} \\
& =\frac{1}{1+\frac{\sigma_{j}\left(\{i, j, 1\}, u_{\{i, j, 1\}}\right)}{\sigma_{i}\left(\{i, j, 1\}, u_{\{i, j, 1\}}\right)}} \\
& =\frac{1}{1+\frac{\sigma_{j}\left(\{i, j\}, u_{\{i, j\}}\right)}{\sigma_{i}\left(\{i, j\}, u_{\{i, j\}}\right)}} \\
& =\frac{\sigma_{i}\left(\{i, j\}, u_{\{i, j\}}\right)}{\sigma_{i}\left(\{i, j\}, u_{\{i, j\}}\right)+\sigma_{j}\left(\{i, j\}, u_{\{i, j\}}\right)} \\
& =\sigma_{i}\left(\{i, j\}, u_{\{i, j\}}\right) .
\end{aligned}
$$

Similarly,

$$
\frac{w_{j}}{w_{i}+w_{j}}=\sigma_{j}\left(\{i, j\}, u_{\{i, j\}}\right) .
$$

Let us denote $\alpha=v(N)-v(i)-v(j)$.

If $\alpha>0$, then CO (for two-person games) together with (13) imply

$$
\begin{aligned}
\sigma_{i}(N, v) & =\sigma_{i}\left(N, \alpha \cdot u_{N}+(v(i), v(j))\right) \\
& =\alpha \cdot \sigma_{i}\left(N, u_{N}\right)+v(i) \\
& =\alpha \cdot \frac{w_{i}}{w_{i}+w_{j}}+v(i) \\
& =S T_{i}^{w}(N, v) .
\end{aligned}
$$


In a similar way, $\mathbf{C O}$ (for two-person games) together with (14) imply

$$
\sigma_{j}(N, v)=S T_{j}^{w}(N, v) .
$$

If $\alpha \leq 0$, the reasoning used in Case 1 applies in this case. Consequently, $\sigma$ satisfies $w-\mathbf{P}$.

The same arguments hold replacing $\mathbf{P C}$ by $\mathbf{S C}$ or $\mathbf{P C}$ by $\mathbf{M C}$ and taking into account Lemma 2 (ii).

From Proposition 2 it turns out that the weighted surplus division solution and the family of weighted Shapley values can be compared by means of consistency together with covariance and path monotonicity.

Theorem 1 Let $\sigma$ be a single-valued solution on $\Gamma$ that satisfies covariance and path monotonicity. Then,

(i) $\sigma$ satisfies projection consistency if and only if there exists a list of positive weights $w \in \mathbb{R}_{++}^{\mathbb{N}}$ such that $\sigma=E S^{w}$.

(ii) $\sigma$ satisfies self consistency if and only if there exists a list of positive weights $w \in \mathbb{R}_{++}^{\mathbb{N}}$ such that $\sigma=S h^{w}$.

Proof

(i) Let $w \in \mathbb{R}_{++}^{\mathbb{N}}$ be a list of positive weights. Clearly, $E S^{w}$ satisfies $\mathbf{C O}$ and PMO (w.r.t. the monotone path $f^{w}$ as defined in (3)). Moreover, Calleja and Llerena (2016) show that it also satisfies PC.

To prove uniqueness, suppose there is a single-valued solution $\sigma$ on $\Gamma$ satisfying these three properties. By Propositions 1 and 2, $\sigma$ satisfies $\mathbf{E}$ and $w-\mathbf{P}$ (w.r.t a list of positive weights $\left.w \in \mathbb{R}_{++}^{\mathbb{N}}\right)$. Let $(N, v)$ be a game. If $|N|=1$, by $\mathbf{E}$ we have $\sigma(N, v)=E S^{w}(N, v)$. If $|N|=2$, by $w-\mathbf{P}$ we have $\sigma(N, v)=E S^{w}(N, v)$. Finally, if $|N| \geq 3$, fix $i \in N$ and take an arbitrary $j \in N \backslash\{i\}$. Let $N^{\prime}=$ $\{i, j\} \subset N$, then,

$$
\begin{aligned}
\sigma_{i}(N, v) & =\sigma_{i}\left(N^{\prime}, r_{P, x}^{N^{\prime}}(v)\right) \\
& =v(i)+\frac{w_{i}}{w_{i}+w_{j}}\left(r_{P, x}^{N^{\prime}}(v)\left(N^{\prime}\right)-v(i)-v(j)\right) \\
& =v(i)+\frac{w_{i}}{w_{i}+w_{j}}\left(\sigma_{i}(N, v)+\sigma_{j}(N, v)-v(i)-v(j)\right),
\end{aligned}
$$

where the first equality follows by $\mathbf{P C}$, the second one by $w-\mathbf{P}$ and the definition of projection reduced game, and the last one by E. Reordering terms, we obtain

$$
\begin{aligned}
\sigma_{i}(N, v)\left(1-\frac{w_{i}}{w_{i}+w_{j}}\right) & =v(i)+\frac{w_{i}}{w_{i}+w_{j}}\left(\sigma_{j}(N, v)-v(i)-v(j)\right) \\
& =v(i)\left(1-\frac{w_{i}}{w_{i}+w_{j}}\right)+\frac{w_{i}}{w_{i}+w_{j}}\left(\sigma_{j}(N, v)-v(j)\right),
\end{aligned}
$$

or, equivalently,

$$
\left(\sigma_{i}(N, v)-v(i)\right) w_{j}=\left(\sigma_{j}(N, v)-v(j)\right) w_{i} .
$$


Notice that this equality holds for all $j \in N \backslash\{i\}$. Adding up,

$$
\left(\sigma_{i}(N, v)-v(i)\right) \sum_{j \in N \backslash\{i\}} w_{j}=w_{i} \sum_{j \in N \backslash\{i\}}\left(\sigma_{j}(N, v)-v(j)\right),
$$

and summing up $\left(\sigma_{i}(N, v)-v(i)\right) w_{i}$ to both sides of the equality we obtain,

$$
\begin{aligned}
\left(\sigma_{i}(N, v)-v(i)\right) \sum_{j \in N} w_{j} & =w_{i} \sum_{j \in N}\left(\sigma_{j}(N, v)-v(j)\right) \\
& =w_{i}\left(v(N)-\sum_{j \in N} v(j)\right),
\end{aligned}
$$

where the last equality follows from E. Hence,

$$
\sigma_{i}(N, v)=v(i)+\frac{w_{i}}{\sum_{j \in N} w_{j}}\left(v(N)-\sum_{i \in N} v(i)\right)=E S_{i}^{w}(N, v) .
$$

(ii) Let $w \in \mathbb{R}_{++}^{\mathbb{N}}$ be a list of positive weights. It is well known that $S h^{w}$ satisfies $\mathbf{S C}$ and CO. In addition, it also satisfies P-MO (w.r.t. the monotone path $f^{w}$ as defined in (3)). Uniqueness comes from Hart and Mas-Colell (1989) (Theorem C) taking into account that P-MO implies monotonicity (by condition (3) in Definition 5) and $\mathbf{C O}$ together with SC imply E (by Proposition 1). ${ }^{7}$

It is well known that the prenucleolus does not satisfy aggregate monotonicity ${ }^{8}$ (and thus path monotonicity). However, imposing this property for two-person games only, we obtain a new characterization of the prenucleolus using max consistency.

Theorem 2 The prenucleolus is the unique single-valued solution on $\Gamma$ that satisfies max consistency, covariance and, for two-person games, path monotonicity.

Proof It is well-known that $\nu_{*}$ satisfies MC, CO and ST, which implies P-MO (w.r.t. the monotone path $\bar{f}$ as defined in (2)) for two-person games.

To prove uniqueness, suppose there is a single-valued solution $\sigma$ on $\Gamma$ satisfying these three properties. By propositions 1 and $2, \sigma$ satisfies $\mathbf{E}$ and $w-\mathbf{P}$. From Hokari (2005) (Proposition 1), E, $w-\mathbf{P}$ and MC jointly imply ST. Finally, since MC and ST imply ETE, by Orshan (1993) (Theorem 3.2) it follows that $\sigma$ coincides with $\nu_{*}$.

Remark 2 The characterizations (and the independence of the properties) stated in Theorem 1 hold if we impose path monotonicity and covariance for two-person games only. Thus, Theorem 1 and Theorem 2 allow for a comparison of the family of weighted Shapley values, the family of weighted surplus division solutions and

\footnotetext{
7 In Hart and Mas-Colell (1989) monotonicity means that everybody is strictly better off whenever the worth of the grand coalition increases and the worth of every other coalition remains unchanged. Notice that monotonicity is a strong version of aggregate monotonicity and weaker that path monotonicity.

8 See, for instance, Hokari (2000).
} 
the prenucleolus by means of consistency. Notice that, although with projection consistency or self consistency together with covariance and path monotonicity (for two-person games) a large family of solutions is characterized, when working with max consistency we obtain only the prenucleolus (not the family of weighted prenucleoli). ${ }^{9}$ The key result is provided by Hokari (2005), who shows that efficiency, w-proportionality and max consistency enforce indeed standardness and thus equal treatment of equals.

Formally, axiomatizations that require some properties for two-person games only are more compelling than those that impose the properties meet for games with an arbitrary number of players. However, axiomatizations that do not force any initial condition for two-person games reflect more accurately the normative behaviour of the solutions. In fact, sometimes we get impossibility results when the properties are required over the full domain of games (see Theorem 5 in Section 4).

\section{Consistency and fairness}

By using fairness, a property due to van den Brink (2001) and related to fairness as introduced by Myerson (1977), instead of path monotonicity, we obtain a new axiomatic comparison of the Shapley value, the equal surplus division solution, the egalitarian non-separable contribution solution and the prenucleolus.

A single-valued solution $\sigma$ on $\Gamma^{\prime} \subseteq \Gamma$ satisfies

- Fairness (F): if for all $(N, v),\left(N, v^{\prime}\right) \in \Gamma^{\prime}$ with $\left(N, v+v^{\prime}\right) \in \Gamma^{\prime}$ and $i, j \in N$ such that $i$ and $j$ are symmetric in $\left(N, v^{\prime}\right)$ we have $\sigma_{i}\left(N, v+v^{\prime}\right)-\sigma_{i}(N, v)=$ $\sigma_{j}\left(N, v+v^{\prime}\right)-\sigma_{j}(N, v)$.

Fairness means that if a game changes by adding another game in which two players $i$ and $j$ are symmetric, then the payoffs of players $i$ and $j$ change by the same amount. If we measure the relevance of a player in terms of marginality, fairness is a quite natural requirement since adding such a game does not change the marginal contributions of symmetric players. Making use of this property, van den Brink (2001) and Casajus (2014) characterize the Shapley value. Not surprisingly, efficiency together with fairness imply path monotonicity. It is not difficult to check that fairness is a self dual property.

Proposition 3 Let $\sigma$ be a single-valued solution on $\Gamma$ that satisfies efficiency and fairness. Then, $\sigma$ satisfies path monotonicity.

Proof Let $\sigma$ be a single-valued solution on $\Gamma$ that satisfies $\mathbf{E}$ and $\mathbf{F}$. Let $N \in \mathcal{N}$, $t \in \mathbb{R}$ and consider two games $(N, v),\left(N, v^{\prime}\right)$ such that $v(S)=v^{\prime}(S)$ for all $S \subset N$ and $v^{\prime}(N)-v(N)=t$. Notice that $v^{\prime}=v+t \cdot u_{N}$. Since all players are symmetric in the game $\left(N, t \cdot u_{N}\right)$, by $\mathbf{F}$ we have $\sigma_{i}\left(N, v^{\prime}\right)-\sigma_{i}(N, v)=\sigma_{j}\left(N, v^{\prime}\right)-\sigma_{j}(N, v)$

\footnotetext{
9 For a formal definition of weighted prenucleoli see, for instance, Derks and Haller (1999).
} 
for all $i, j \in N$. Finally, by $\mathbf{E}$ we obtain $\sigma_{i}\left(N, v^{\prime}\right)-\sigma_{i}(N, v)=\frac{t}{|N|}$ for all $i \in N$. Hence, $\sigma$ satisfies P-MO (w.r.t. the monotone path $\bar{f}$ as defined in (2)).

Proposition 3 shows that, under efficiency, path monotonicity can be viewed as a relaxation of fairness by allowing that when the worth of the grand coalition varies, the players' payoffs change in the same direction, but not necessarily by the same amount, as fairness suggests. Now, replacing path monotonicity by fairness in Theorem 1, we obtain new axiomatic characterizations of the Shapley value and the equal surplus division solution. Moreover, imposing complement consistency we provide a new axiomatic characterization of the egalitarian non-separable contribution solution.

Theorem 3 Let $\sigma$ be a single-valued solution on $\Gamma$ that satisfies covariance and fairness. Then,

(i) $\sigma$ satisfies projection consistency if and only if it coincides with the equal surplus division solution.

(ii) $\sigma$ satisfies self consistency if and only if it coincides with the Shapley value.

(iii) $\sigma$ satisfies complement consistency if and only if it coincides with the egalitarian non-separable contribution solution.

Proof

(i) It is clear that the equal surplus division solution satisfies $\mathbf{P C}, \mathbf{F}$ and $\mathbf{C O}$. Let $\sigma$ be a single-valued solution on $\Gamma$ that satisfies these three properties. By Proposition 1 and Proposition 3, $\sigma$ obeys $\mathbf{E}$ and P-MO (w.r.t. the monotone path $\bar{f}$ ). Now uniqueness comes following the proof of Theorem 1 (i) taking into account that all players have the same weight.

(ii) It is well known that the Shapley value satisfies $\mathbf{S C}, \mathbf{F}$ and $\mathbf{C O}$. Let $\sigma$ be a single-valued solution on $\Gamma$ that satisfies these three properties. By Proposition 1 and Proposition 3, $\sigma$ obeys $\mathbf{E}$ and P-MO (w.r.t. the monotone path $\bar{f}$ ). It is not difficult to check that CO, E and P-MO (w.r.t. $\bar{f}$ ) imply ST. Now uniqueness comes from Hart and Mas-Colell (1989) (Theorem B).

(iii) Clearly, the egalitarian non-separable contribution solution satisfies $\mathbf{C C}, \mathbf{F}$ and CO. Let $\sigma$ be a single-valued solution on $\Gamma$ satisfying these three properties. The dual solution $\sigma^{d}$ satisfies $\mathbf{P C}, \mathbf{F}$ and $\mathbf{C O}$ since $\mathbf{C C}$ and $\mathbf{P C}$ are dual to each other and $\mathbf{C O}$ and $\mathbf{F}$ are self dual properties. Thus, by statement (i), $\sigma^{d}=E S$. In view of the fact that the egalitarian non-separable contribution solution is dual to the equal surplus division solution, we conclude that $\sigma=$ ENSC.

Since the prenucleolus satisfies fairness for two-person games (because it satisfies standarness), from Proposition 1, Proposition 3 and Theorem 2 we obtain an alternative characterization.

Theorem 4 The prenucleolus is the unique single-valued solution on $\Gamma$ that satisfies max consistency, covariance and, for two-person games, fairness. 
Remark 3 The characterizations stated in Theorem 3 hold if we impose fairness for two-person games only. Thus, Theorem 3 and Theorem 4 allow for a comparison of the Shapley value, the equal surplus division solution, the egalitarian non-separable contribution solution and the prenucleolus by means of consistency.

Since the prenucleolus does not satisfy neither path monotonicity nor fairness (see Proposition 3) for games with an arbitrary number of players, two impossibility results emerge from both Theorem 2 and Theorem 4.

Theorem 5 There is no single-valued solution on $\Gamma$ that satisfies

(i) max consistency, covariance and path monotonicity.

(ii) max consistency, covariance and fairness.

\section{Concluding remarks}

Theorem C in Hart and Mas-Colell (1989) characterizes the family of weighted Shapley values by means of self consistency and, for two-person games, efficiency, covariance and monotonicity. Nevertheless, from Proposition 1 it comes out that efficiency can be dropped from their characterization result. Thus, it can be reformulated as follows:

Theorem 6 A single-valued solution $\sigma$ on $\Gamma$ satisfies self consistency and, for two-person games, covariance and monotonicity if and only if there exists a list of positive weights $w \in \mathbb{R}_{++}$such that $\sigma=S h^{w}$.

This reformulation opens an interesting question: which is the set of rules that emerges from substituting in Theorem 6 self consistency by either projection consistency, complement consistency or max consistency? Notice that we partially overcome this problem working with path monotonicity. Unfortunately, we have been unsuccessful in our attempts to solve this problem using monotonicity as defined in Hart and Mas-Colell (1989), and we leave it for future research.

It is well known that efficiency, covariance and equal treatment of equals for two-person games are equivalent to standardness. The Shapley value, the equal surplus division solution and the egalitarian non-separable contribution solution satisfy these properties for any game. Moreover, covariance only for two-person games together with either self consistency, projection consistency or complement consistency imply efficiency (Proposition 1). Consequently, Theorem B' in Hart and Mas-Colell (1989) can be reformulated (dropping efficiency) in terms of covariance and equal treatment of equals for two-person games, together with self consistency. Moreover, Corollary 4.4. (i) in Driessen and Funaki (1997) can be rewritten by means of covariance and equal treatment of equals together with projection consistency, dropping efficiency too. Finally, the egalitarian non-separable contribution solution can be characterized by means of covariance, equal treatment of equals and complement consistency, which is also a refinement of Corollary 
4.4. (ii) in Driessen and Funaki (1997). ${ }^{10}$ Interestingly, Sobolev (1975) characterizes the prenucleolus as the unique single-valued solution that satisfies covariance, anonymity $^{11}$ and max consistency. Orshan (1993) shows that anonymity can be weakened and replaced by equal treatment of equals. Theorem 2, Theorem 3 and Theorem 4 modify, respectively, these characterizations replacing equal treatment of equals by path monotonicity/fairness (for two-person games). It is not difficult to check that path monotonicity/fairness neither imply equal treatment of equals nor they are implied by it.

As underlined in Section 3, not all weighted egalitarian non-separable contribution solution, ENSC ${ }^{w}$, satisfy aggregate monotonicity (see Example 2). Therefore, complement consistency, covariance and path monotonicity do not characterize the family of $E N S C^{w}$. However, for any list of positive weights, the $E N S C^{w}$ meets path monotonicity for two-person games. ${ }^{12}$ In view of Theorem 2 , one may wonder if complement consistency, covariance and, for two-person games, path monotonicity, characterize the family of $E N S C^{w}$. At this point some difficulties appear. First, as the next example shows, $E N S C^{w}$ does not satisfy $w$-proportionality. Let $(N, v)$ be a game with $N=\{1,2\}, v(1)=1, v(2)=0, v(N)=2$ and consider $w \in \mathbb{R}_{++}^{\mathbb{N}}$ such that $w_{1}=2$ and $w_{2}=1$. Then, $\operatorname{ENSC}^{w}(N, v)=\left(\frac{4}{3}, \frac{2}{3}\right)$ and $S T^{w}(N, v)=\left(\frac{5}{3}, \frac{1}{3}\right)$. Thus, Proposition 2 does not hold when imposing complement consistency. If we want to use the duality approach from Theorem 1(i) (and taking into account Remark 2), a second drawback is that path monotonicity for two-person games is neither self dual. To see it, consider the single-valued solution $\varrho$ defined, for all $N \in \mathcal{N}$ and all $(N, v) \in \Gamma$, as follows:

$$
\varrho(N, v):= \begin{cases}E S(N, v) & \text { if }|N| \neq 2, \\ \left(v(i)+\frac{1}{2} v(N), \frac{1}{2} v(N)-v(i)\right) & \text { if } N=\{i, j\} .\end{cases}
$$

Notice that $\varrho$ satisfies path monotonicity (w.r.f. $\bar{f}$ as defined in (2)). The dual solution $\varrho^{d}$ is given by setting, for all $N \in \mathcal{N}$ and all $(N, v) \in \Gamma$,

$$
\varrho^{d}(N, v):= \begin{cases}E N S C(N, v) & \text { if }|N| \neq 2, \\ \left(\frac{3}{2} v(N)-v(j), v(j)-\frac{1}{2} v(N)\right) & \text { if } N=\{i, j\} .\end{cases}
$$

Let $(N, v),\left(N, v^{\prime}\right)$ be two games with set of players $N=\{1,2\}$ and characteristic functions: $v(i)=v^{\prime}(i)=0$ for all $i \in N, v(N)=1$ and $v^{\prime}(N)=2$. Then, $\varrho^{d}(N, v)=\left(\frac{3}{2},-\frac{1}{2}\right)$ and $\varrho^{d}\left(N, v^{\prime}\right)=(3,-1)$, which proves that $\varrho^{d}$ is not aggregate monotonic, and hence path monotonicity is not self dual for two-person games. Thus, in future research it could be interesting to pay attention to this issue.

Nevertheless, a characterization of the family of weighted non-separable contribution solutions can be derived introducing the dual property of path monotonicity.

\footnotetext{
10 In Driessen and Funaki (1997), efficiency is included in their definition of single-valued solution.

11 In words, anonymity simply says that the solution is independent of the names of the players (see Peleg and Sudhölter, 2007, for a formal definition).

12 Let $w \in \mathbb{R}_{++}^{\mathbb{N}}$. It can be easily checked that $E N S C^{w}$ satisfies, for two-person games, path monotonicity w.r.t. a monotone path $g^{w} \in \mathcal{F}_{\text {mon }}$ such that, for all $N=\{i, j\} \in \mathcal{N}$ and all $t \in \mathbb{R}, g_{i}^{w}(N, t)=\frac{w_{j}}{w_{i}+w_{j}} \cdot t$ and $g_{j}^{w}(N, t)=\frac{w_{i}}{w_{i}+w_{j}} \cdot t$. Notice that $g^{w} \neq f^{w}$ as defined in (3).
} 
A single-valued solution $\sigma$ on $\Gamma^{\prime} \subseteq \Gamma$ satisfies

- Constant shift path monotonicity: ${ }^{13}$ if there exists a monotone path $f \in$ $\mathcal{F}_{\text {mon }}$ such that, for all $N \in \mathcal{N}$ and all $(N, v),\left(N, v^{\prime}\right) \in \Gamma^{\prime}$ with $v^{\prime}(N)-v(N)=$ $v^{\prime}(S)-v(S)$ for all $\emptyset \neq S \subset N$, it holds $\sigma\left(N, v^{\prime}\right)-\sigma(N, v)=f\left(N, v^{\prime}(N)-v(N)\right)$.

Constant shift path monotonicity says that if the impact in the game $(N, v)$ is such that all coalitions increase (decrease) in the same amount $t$ and players agree on how to distribute it, then they should respect this agreement regardless of the initial game $(N, v)$. It is not difficult to check that constant shift path monotonicity and path monotonicity are dual to each other. This fact, together with Theorem 1 (i), lead to the following characterization result.

Theorem 7 A single-valued solution $\sigma$ on $\Gamma$ satisfies complement consistency, covariance and constant shift path monotonicity if and only if there exists a list of positive weights $w \in \mathbb{R}_{++}^{\mathbb{N}}$ such that $\sigma=E N S C^{w}$.

To finish, a quite natural question is to study the implications of considering weakly monotonic functions rather than strictly monotonic functions in the definition of a monotone path (Definition 5). It turns out that imposing "weak" path monotonicity in Theorem 1 (i) and (ii) provides larger classes of single-valued solutions than $E S^{w}$ and $S h^{w}$ that include, for instance, any marginal contribution solution and any $f^{\pi}$ - surplus division solution (see Appendix for formal definitions), respectively. On the other hand, the single-valued solution introduced by Peleg and Sudhöter (2007) (see Section 6.3.2. p.118) satisfies, on the domain of balanced games, ${ }^{14}$ covariance, max consistency and, for two-person games, "weak" path monotonicity. Consequently, on balanced games, imposing "weak" path monotonicity in Theorem 2 does not characterize the prenucleolus. This may suggest that, on the full domain of games, Theorem 2 does not remain valid when working with this weaker form of path monotonicity, although it is still an open question. In our opinion, dealing with such problems may require a good understanding of weighted solutions and properties when zero weights are allowed, in the line of the works of Kalai and Samet (1987), Monderer, Samet and Shapley (1992) or Nowak and Radzik (1995) for the weighted Shapley values, or Hokari (2005) for the prenucleolus.

\section{Appendix}

This appendix contains the independence of the properties used in the characterization results. To do this, let us first introduce the following single-valued solutions:

\footnotetext{
13 We thank an anonymous referee for providing us this property.

14 A game $(N, v)$ is said to be balanced if $C(N, v)=\{x \in X(N, v) \mid x(S) \geq v(S)$ for all $S \subseteq$ $N\} \neq \emptyset$.
} 
1. The equal divison solution, denoted by $E D$, is defined as follows: for all $N \in \mathcal{N}$, all $(N, v) \in \Gamma$ and all $i \in N$,

$$
E D_{i}(N, v):=\frac{v(N)}{n} .
$$

2. Let $\pi$ be a permutation on $\mathbb{N}$, the marginal contribution solution relative to $\pi$, denoted by $m c^{\pi}$, is defined as follows: for all $N \in \mathcal{N}$, all $(N, v) \in \Gamma$ and all $i \in N$

$$
m c_{i}^{\pi}(N, v):=v(\{j \in N \mid \pi(j) \leq \pi(i)\})-v(\{j \in N \mid \pi(j)<\pi(i)\}) .
$$

3. Let $\pi$ be a permutation on $\mathbb{N}$, the $f^{\pi}$-surplus division solution, denoted by $E S^{f^{\pi}}$, is defined as follows: for all $N \in \mathcal{N}$, all $(N, v) \in \Gamma$ and all $i \in N$,

$$
E S_{i}^{f^{\pi}}(N, v):=v(i)+f_{i}^{\pi}\left(N, v(N)-\sum_{i \in N} v(i)\right),
$$

where $f^{\pi}$ is defined as follows: for all $t \in \mathbb{R}, f^{\pi}(N, t)=t \cdot e_{\{j\}}$, being $j \in N$ such that $\pi(j) \geq \pi(i)$ for all $i \in N$. $f^{\pi}$ assigns all the amount $t$ to the last player in $N$ according to $\pi$.

- Independence of the properties in Theorem 1, Theorem 3, Theorem 6 and Theorem 7:

$E S^{f^{\pi}}$ satisfies covariance and projection consistency but, for two-person games, neither monotonicity (and thus path monotonicity) nor fairness. The dual solution of $E S^{f^{\pi}}$ satisfies covariance and complement consistency but neither fairness nor constant shift path monotonicity. Sh satisfies covariance, path monotonicity, constant shift path monotonicity and fairness but neither projection consistency nor complement consistency. ED satisfies path monotonicity, constant shift path monotonicity, fairness, projection consistency, complement consistency and self consistency but not covariance for two-person games. Additionally, the marginal contribution solution, $m c^{\pi}$, satisfies covariance and self consistency but, for two-person games, neither monotonicity nor fairness. ES satisfies covariance, path monotonicity and fairness but not self consistency.

- Independence of the properties in Theorem 2 and Theorem 4: ED satisfies max consistency, path monotonicity and fairness, but not covariance. ES satisfies path monotonicity, fairness and covariance, but not max consistency. Finally, to find a single-valued solution satisfying max consistency and covariance but, for two-person games, neither path monotonicity nor fairness, see Lemma 6.3.15 in Peleg and Sudhölter (2007).

\section{Acknowledgements}

The authors thank two anonymous referees and an associated editor for helpful and inspiring comments. We also acknowledge the support from research grant ECO2016-75410-P (AEI/FEDER, UE) and ECO2017-86481-P (AEI/FEDER, UE) (Ministerio de Economía y Competitividad). 


\section{References}

1. Béal, S., E. Rémila and P. Solal (2014). Axioms of invariance for TU-games. International Journal of Game Theory, 44:891-902.

2. Calleja, P. and F. Llerena (2016). Rationality, aggregate monotonicity and consistency in cooperative games: some (im)possibility results. Social Choice and Welfare, 48:197-220.

3. Casajus, A. (2011). Differential marginality, van den Brink fairness, and the Shapley value. Theory and Decision, 71:163-174.

4. Casajus, A. (2014). The Shapley value without efficiency and additivity. Mathematical Social Sciences, 68:1-4.

5. Casajus, A. and F. Huettner (2014). Null, nullifying, or dummifying players: The difference between the Shapley value, the equal division value, and the equal surplus division value. Economics Letters, 12: 167-169.

6. Chun, Y. and B. Park (2012). Population solidarity, population fair-ranking, and the egalitarian value. International Journal of Game Theory, 41: 255-270.

7. Davis, M. and M. Maschler (1965). The kernel of a cooperative game. Naval Research Logistics Quarterly, 12: 223-259.

8. Derks, J.J.M. and H. Haller (1999). Weighted nucleoli. International Journal of Game Theory, 28: 173-187.

9. Driessen, T. and Y. Funaki (1991). Coincidence of and collinearity between game theoretic solutions. OR Spektrum, 13: 15-30.

10. Driessen, T. and Y. Funaki (1997). Reduced game properties of egalitarian division rules for TU-games. In: Parthasarathy et al. (eds.) Game theoretical applications to economics and operations research. Kluwer Academic Publishers, Netherlands, 85-103.

11. Funaki, Y. (1998). Dual axiomatizations of solutions of cooperative games. Mimeo.

12. Hart S. and A. Mas-Colell (1989). Potential, Value, and Consistency. Econometrica, 57: 589-614.

13. Hokari, T. (2000). The nucleolus is not aggregate-monotonic on the domain of convex games. International Journal of Game Theory, 29: 133-137.

14. Hokari, T. (2005). Consistency implies equal treatment in TU-games. Games and Economic Behavior, 51:63-82.

15. Hwang, Y.-A. (2006). Associated Consistency and Equal Allocation of Nonseparable Costs. Economic Theory, 28:709-719.

16. Jung S-M. (2011). Hyers-Ulam-Rassias Stability of Functional Equations in Nonlinear Analysis. Springer, New York Dordrecht Heidelberg London.

17. Kalai, E. (1977). Proportional Solutions to Bargaining Situations: Interpersonal Utility Comparisons. Econometrica, 45:1623-1630.

18. Kalai, E. and D. Samet (1985). Monotonic Solutions to General Cooperative Games. Econometrica, 53:307-327.

19. Kalai, E and D. Samet (1987). On weighted Shapley values. International Journal of Game Theory, 16: 205-222.

20. Kalai, E. and M. Smorodinsky (1975). Other solutions to Nash's Bargaining Problems. Econometrica, 43:513-518.

21. Megiddo, N. (1974). On the nonmonotonicity of the bargaining set, the kernel and the nucleolus of a game. SIAM J. Appl Math, 27: 355-358.

22. Monderer, D., Samet, D. and L.S. Shapley (1992). Weighted values and the Core. International Journal of Game Theory, 21: 27-39.

23. Moulin, H. (1985). The separability axiom and equal sharing methods. Journal of Economic Theory, 36: 120-148.

24. Myerson, RB. (1977). Graphs and cooperation in games. Mathematics of Operations Research, 2:225-229.

25. Nowak, A.S. and T. Radzik (1995). On axiomatizations of the weighted Shapley values. Games and Economic Behavior, 8:389-405.

26. Oishi, M., Nakayama, T., Hokari T. and Y. Funaki (2016). Duality and anti-duality in TU games applied to solutions, axioms, and axiomatizations. Journal of Mathematical Economics, 63: 44-53. 
27. Orshan, G. (1993). The prenucleolus and the reduced game property: equal treatment replaces anonymity. International Journal of Game Theory, 22: 241-248.

28. Peleg, B. and P. Sudhölter (2007). Introduction of the Theory of Cooperative Games. Springer-Verlag, Berlin Heidelberg.

29. Schmeidler, D. (1969). The nucleolus of a characteristic function game. SIAM Journal Appl Math, 17: 1163-1170.

30. Shapley, L.S. (1953 a). Additive and non-additive set functions. Ph.D. Thesis, Princeton University.

31. Shapley, L.S. (1953 b). A value for n-person games. In: Kuhn HW, Tucker NW (eds.) Contributions to the theory of games. Princeton University Press, Princeton, 307-317.

32. Sobolev, A.J. (1975). The characterization of optimality principles in cooperative games by functional equations. In: Mathematical Methods in the Social Sciences, ed. by N.N. Vorobiev, Vol. 6, pp 95-151, Vilnius. Academy of Sciences of the Lithuanian SSR, in Russian.

33. Thomson, W. (1987). Monotonicity of bargaining solutions with respect to the disagreement point. Journal of Economic Theory, 42:50-58.

34. Thomson, W. (2003). Consistent allocation rules. Mimeo, University of Rochester.

35. Thomson, W. (2011). Consistency and its converse: an introduction. Review of Economic Design, 15: 257-291.

36. Thomson, W. (2012). On the axiomatics of resource allocation: interpreting the consistency principle. Economics and Philosophy, 28: 385-421.

37. van den Brink, R. (2001). An axiomatization of the Shapley value using a fairness property. International Journal of Game Theory, 30: 309-319.

38. van den Brink, R. (2007). Null or nullifying players: the difference between the Shapley value and equal division solutions. Journal of Economic Theory, 136: 767-775.

39. van den Brink, R., Chun, Y., Funaki, Y. and B. Park (2016). Consistency, population solidarity, and egalitarian solutions for TU-games. Theory and Decision, 81: 427-447.

40. Young, H.P. (1985). Monotonic solutions of cooperative games, International Journal of Game Theory 14 (1985) 65-72. 